Pacific Journal of Mathematics

AN EXPLICIT FORMULA FOR THE FUNDAMENTAL UNITS
OF A REAL PURE SEXTIC NUMBER FIELD AND ITS GALOIS
CLOSURE Ken NAKAMU 


\title{
AN EXPLICIT FORMULA FOR THE FUNDAMENTAL UNITS OF A REAL PURE SEXTIC NUMBER FIELD AND ITS GALOIS CLOSURE
}

\author{
KEN NAKAMULA
}

The object of this paper is to give a set of fundamental units of a real pure sextic number field $K=Q\left(\sqrt[6]{a^{6}-1}\right)$, where $a$ is a special type of natural number and $a^{b}-1$ is not necessarily 6 th power free. It is also shown that a set of fundamental units of the galois closure $L=K(\sqrt{-3)}$ of $K$ is formed by a real unit and its conjugates.

Let $d$ be a 6 th power free natural number which is not a perfect

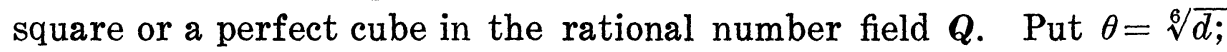
then $K=\boldsymbol{Q}(\theta)$ is a real pure sextic number field. We investigate the group of units of $K$ for a special type of $d$ as follows. Let $d$ be given by

$$
d=c\left(b^{6} c \pm 2\right)\left(b^{12} c^{2} \pm b^{6} c+1\right)\left(b^{12} c^{2} \pm 3 b^{6} c+3\right)
$$

with natural numbers $b$ and $c$. Put

$$
a=b^{6} c \pm 1 \text {. }
$$

(The \pm signs correspond respectively throughout this paper.) Then

$$
b^{6} d=a^{6}-1
$$

and $K=Q\left(\sqrt[6]{a^{6}-1}\right)$.

THEOREM 1. The notation being as above, we assume that $d>1$ and $d$ is square free. Then

$$
\xi_{1}=a-b \theta, \quad \xi_{2}=a+b \theta, \quad \xi_{3}=a^{2}+a b \theta+b^{2} \theta^{2}
$$

form a set of fundamental units of $K$.

As to explicit formulas for the fundamental units of number fields, G. Degert [2] has given one for certain real quadratic fields. As an application of the Jacobi-Perron algorithm (J.P.A.), L. Bernstein, H.-J. Stender and R. J. Rudman has extended Degert's result to certain real pure cubic, quartic and sextic fields (see [9] and [10]). On the other hand, $H$. Yokoi has given a different formula for the fundamental units of real quadratic and pure cubic number fields in [11], [12] and [13]. Theorem 1 is an extension of Yokoi's result to real pure sextic fields. A similar formula can be 
obtained for the fundamental units of real pure quartic fields (see [7]). Theorem 1 is not included in Stender's result when $b>1$ (see Remark 4).

THEOREM 2. Under the same assumption as in Theorem 1, any 5 of 6 conjugates of $\xi_{1}$ form a set of fundamental units of $K(\sqrt{-3})$.

Theorem 2 gives an example of a real Minkowski unit of a non-abelian galois extension $K(\sqrt{-3}) / Q$ (see [1]).

To prove Theorem 1, we use the same method as in Stender [8]. Let $K_{2}$ and $K_{3}$ be the quadratic land 'cubic subfields of $K$ respectively, and let $E$ be the group of units of $K$. Define the group $H$ of positive relative units of $K$ with respect to $K_{2}$ and $K_{3}$ by

$$
H=\left\{\xi \in E \mid N_{2}(\xi)=N_{3}(\xi)=1\right\},
$$

where $N_{2}$ and $N_{3}$ denote the relative norm maps from $K$ to $K_{2}$ and $K_{3}$ respectively. Then $H$ is a free abelian group of rank 1 . The fundamental units of the subfields will be determined in $\S 1$. A generator of $H$ will be determined in $\S 2$. In $\S 3$, we shall prove Theorem 1 and show the existence of infinitely many fields which satisfy the condition of Theorem 1 . In $\S 4$, we shall prove Theorem 2 .

The author wishes to thank Prof. H. Yokoi for his advice during the preparation of the manuscript, and Prof. H.-J. Stender for sending a copy of his paper [10] in manuscript.

1. Fundamental units of the subfields. Let $d$ be a natural number given by (1) with natural numbers $b$ and $c$, and define $a$ as in (2). Assume that $d$ is neither a perfect square nor a perfect cube in $\boldsymbol{Q}$. Then $K=\boldsymbol{Q}(\theta)$, where $\theta=\sqrt[6]{d}$, is of degree 6 over $\boldsymbol{Q}$, and it contains the quadratic subfield $K_{2}=Q\left(\theta^{3}\right)$ and the cubic subfield $K_{3}=$ $Q\left(\theta^{2}\right)$. Denote respectively by $\eta_{2}$ and $\eta_{3}$ the fundamental units of $K_{2}$ and $K_{3}$ which are larger than 1 . Define the algebraic integers $\xi_{1}, \xi_{2}, \xi_{3}$ as in (4). Then it immediately follows from (3) that their absolute norms are all equal to 1 ; hence they belong to the group $E$ of units of $K$. We also see that $1 / \xi_{1} \xi_{3}=a^{3}+b^{3} \theta^{3}$ belongs to $E \cap K_{2}$, and that $1 / \xi_{1} \xi_{2}=a^{4}+a^{2} b^{2} \theta^{2}+b^{4} \theta^{4}$ belongs to $E \cap K_{3}$.

Proposition 1. If $d>1$ and is square free, then $\eta_{2}=1 / \xi_{1} \xi_{3}=$ $a^{3}+b^{3} \theta^{3}$.

Proof. Since $1 / \xi_{1} \xi_{3}>1$, we have $\eta_{2}^{n}=a^{3}+b^{3} \theta^{3}$ with a natural number $n$. Let us assume $n \geqq 2$. We can write $\eta_{2}=\left(t+u \theta^{3}\right) / 2$ 
with nonzero rational integers $t$ and $u$, because $d$ is square free. Then $u=\left(\eta_{2}-\eta_{2}^{\prime}\right) / \theta^{3}$, where $\eta_{2}^{\prime}=\left(t-u \theta^{3}\right) / 2$. Taking into account that $u \neq 0,\left|\eta_{2}^{\prime}\right|=1 / \eta_{2}<1, n \geqq 2$ and $a^{3}+b^{3} \theta^{3}>1$, we see that

$$
1 \leqq|u| \leqq\left(\eta_{2}+\left|\eta_{2}^{\prime}\right|\right) / \theta^{3}<\sqrt{\left(a^{3}+b^{3} \theta^{3}\right) / d}+\sqrt{1 / d} .
$$

From (3), $b \theta<a$ and $1 / d=b^{6} /\left(a^{6}-1\right)$. Therefore

$$
1<\sqrt{2 a^{3} b^{6} /\left(a^{6}-1\right)}+\sqrt{b^{6} /\left(a^{6}-1\right)} .
$$

From $(2), b^{6} \leqq a+1$. Then

$$
1<\sqrt{2 a^{3}(a+1) /\left(a^{6}-1\right)}+\sqrt{(a+1) /\left(a^{6}-1\right)} .
$$

However, the right side of the last inequality is smaller than 1 for $a \geqq 3$, which is a contradiction. When $a=2$, we see from (3) that $b=1$, and then $d=63$ is not square free. Since $a \geqq 2$ by (3), $n=1$ under our assumption, and the proposition follows.

REMARK 1. When $d$ has a square factor, the conclusion of Proposition 1 does not necessarily hold. For example, set $b=1$ and $c=22$ in (1) and (2) for the plus case, i.e.,

$$
d=22(22+2)\left(22^{2}+22+1\right)\left(22^{2}+3 \cdot 22+3\right), \quad a=22+1 .
$$

Then $d=2^{4} \cdot 3^{2} \cdot 7 \cdot 11 \cdot 13^{2} \cdot 79, a=23$, and

$$
\eta_{2}=2 \cdot 3 \cdot 13+\sqrt{7 \cdot 11 \cdot 79}, \eta_{2}^{2}=a^{3}+b^{3} \theta^{3} .
$$

When the square factor of $d$ is small, Proposition 1 is also true as is seen from the proof.

Proposition 2. If $d>1$ and is cube free, then $\eta_{3}=1 / \xi_{1} \xi_{2}=$ $a^{4}+a^{2} b^{2} \theta^{2}+b^{4} \theta^{4}$.

Proof. It follows from T. Nagell [5] (see also [13]), that the binomial unit $\xi_{1} \xi_{2}=a^{2}-b^{2} \theta^{2}$ is either fundamental unit of $K_{3}$ or its square, and the latter occurs only for $d=20,50$ and a finite number of $d \equiv \pm 1(\bmod 9)$. Now we assume $1 / \eta_{3}^{2}=a^{2}-b^{2} \theta^{2}$. Let $d=f g^{2}$ with relatively prime natural numbers $f$ and $g$, and write $1 / \eta_{3}=$ $\left\{x+y \theta^{2}+(z / g) \theta^{4}\right\} / 3$ with rational integers $x, y$ and $z$. Then

$$
|y|<\left\{1+2 \sqrt[4]{1\left(a^{2}-b^{2} \theta^{2}\right)}\right\} / \theta^{2}
$$

follows similarly as in [5]. Note $I, 1$. Here

$$
1 /\left(a^{2}-b^{2} \theta^{2}\right)=a^{4}+a^{2} b^{2} \theta^{2}+b^{4} \theta^{4}<3 a^{4}
$$

and 


$$
1 / \theta^{2}=\sqrt[3]{1 / d}=\sqrt[3]{b^{6} /\left(a^{6}-1\right)} \leqq \sqrt[3]{(a+1) /\left(a^{6}-1\right)}
$$

are obtained as before, and hence

$$
|y|<\sqrt[3]{(a+1) /\left(a^{6}-1\right)}+2 \sqrt[4]{3} \sqrt[3]{a^{3}(a+1) /\left(a^{6}-1\right)} .
$$

When $a \geqq 6$, the right side of the last inequality is smaller than 1 . Therefore $y=0$ and $1 / \eta_{3}=\left\{x+(z / g) \theta^{4}\right\} / 3$. This is a contradiction, because the square of a binomial unit cannot be binomial. When $a=2,3,4$ or $5, a^{6}-1$ is 6 th power free, and, by (3), $b=1$ and $d=a^{6}-1$. For $a=2,4$ or 5 , we have $d \equiv 0 \not \equiv \pm 1(\bmod 9)$. For $a=3$, we see that $d$ is not cube free. This completes the proof.

REMARK 2. By the same method as in the proof of Proposition 2, we can verify that the exceptional case of Theorem 6(iii) of [10] occurs only when $(u, n)=(1,4)$, i.e., $d=28$.

REMARK 3. As we have seen in the end of the proof of Proposition 2 , we have $a \geqq 6$ when $b \geqq 2$. This fact will be used in the next section.

2. Relative fundamental unit. Let $d, a$ and $K$ be as in $\S 1$. We keep the notation as before. Let $H$ be the group of positive relative units of $K$ with respect to $K_{2}$ and $K_{3}$ which is defined by (5). Then, as in [8], $11, \mathrm{II}, 8$, the group $H$ is a free abelian group of rank 1. We denote by $\varepsilon_{1}$ the generator of $H$ which is larger than 1 . Suppose $d>1$ and is square free. Then, by Propositions 1 and 2,

$$
\eta_{2}=1 / \xi_{1} \xi_{3}=a^{3}+b^{3} \theta^{3}, \quad \eta_{3}=1 / \xi_{1} \xi_{2}=a^{4}+a^{2} b^{2} \theta^{2}+b^{4} \theta^{4} .
$$

The field belongs to Klasse $I$ of [8], because

$$
N_{2}\left(1 / \xi_{1}\right)=\eta_{2}, \quad N_{3}\left(1 / \xi_{1}\right)=\eta_{3} .
$$

Put now $\varepsilon=1 / \xi_{1}^{8} \eta_{2}^{2} \eta_{3}^{3}$, then $\varepsilon \in H$ and

$$
\varepsilon=\xi_{2}^{3} \xi_{3}^{2} / \xi_{1}=(a+b \theta)^{3}\left(a^{2}+a b \theta+b^{2} \theta^{2}\right)^{2}\left(a^{5}+a^{4} b \theta+\cdots+b^{5} \theta^{5}\right)
$$

by (3) and (6).

Proposition 3. If $d>1$ and is square free, then $\varepsilon_{1}=\varepsilon=\xi_{2}^{3} \xi_{3}^{2} / \xi_{1}$.

Proof. When $b=1, d=a^{6}-1$ by (3), and then Stender has shown that $\varepsilon_{1}=\varepsilon$ in [8], Hilfssatz 7. Let $b \geqq 2$. Since $\varepsilon>1$ and $\varepsilon \in H, \varepsilon_{1}^{n}=\varepsilon$ with a natural number $n$. Assume $n \geqq 2$. The relative unit $\varepsilon=1 / \xi_{1}^{6} \eta_{2}^{2} \eta_{3}^{3}$ can be neither a square nor a cube in $K$ by [8], Hilfssatz 1 . Therefore $n \geqq 5$. Now we can write $\varepsilon_{1}=1 / 6 \sum_{j=0}^{5} x_{j} \theta^{j-1}$ 
with rational integers $x_{j}(j=0,1, \cdots, 5)$ according to [8], Hilfssatz 2. Note that $d$ divides $x_{0}$ and that either $x_{0}$ or $x_{5}$ is distinct from zero by [9], Hilfssatz 3. On the other hand, by [8], (1.6),

$$
\left|x_{j}\right|<\theta^{1-j} A(j=0,1, \cdots, 5) \text { with } A=\sqrt[5]{\varepsilon}+2 \sqrt[10]{\varepsilon}+3,
$$

since $n \geqq 5$ and $\varepsilon>1$. Hence either

$$
d=\theta^{6} \leqq\left|x_{0}\right|<\theta A
$$

or

$$
1 \leqq\left|x_{5}\right|<A / \theta^{4}
$$

should hold. From the fact that $\theta>1$, we obtain

$$
1<A / \theta^{4}=A \sqrt[3]{1 / d^{2}} .
$$

Taking into account that $b \theta<a$ and $1 / d=b^{6} /\left(a^{6}-1\right) \leqq(a+1) /\left(a^{6}-1\right)$ as before, we can derive

$$
1<\sqrt[3]{(a+1)^{2} /\left(a^{6}-1\right)^{2}}\left(\sqrt[5]{2^{4}} \cdot 3^{3} a^{12}+2 \sqrt[10]{2^{4} \cdot 3^{3} a^{12}}+3\right) .
$$

However, since $a \geqq 6$ as we have mentioned in Remark 3 , the right side of the last inequality is smaller than 1 . This is a contradiction. Thus $\varepsilon_{1}=\varepsilon$ for $b \geqq 2$, too.

3. Fundamental units of $K$. For natural numbers $b$ and $c$, let $d$ and $a$ be given by (1) and (2). Let $K=\boldsymbol{Q}(\theta)$, where $\theta=\sqrt[6]{d}$. Further let $\xi_{1}, \xi_{2}, \xi_{3}$ be given by (4).

THeOREM 1. (i) If $d>1$ and is square free, then $\xi_{1}, \xi_{2}, \xi_{3}$ form $a$ set of fundamental units of $K$.

(ii) For a fixed natural number b, there are infinitely many values of $c$ which make $d$ square free.

Proof. (i) Recall that $K$ belongs to Klasse $I$ of [8] by (7). It follows from Propositions 1, 2 and 3 that

$$
\varepsilon_{1}=\xi_{2}^{3} \xi_{3}^{2} / \xi_{1}, \quad \sqrt[3]{\eta_{2} / \varepsilon_{1}}=1 / \xi_{2} \xi_{3}, \quad \sqrt{\eta_{3} \varepsilon_{1}}=\xi_{2} \xi_{3} / \xi_{1} .
$$

These three units form a set of fundamental units of $K$ by [8], Satz $1^{\prime}$. Hence the assertion is obvious.

(ii) Let

$$
f(X)=X\left(b^{6} X \pm 2\right)\left(b^{12} X^{2} \pm b^{6} X+1\right)\left(b^{12} X^{2} \pm 3 b^{6} X+3\right) .
$$

We shall find infinitely many square free natural numbers in the sequence $\{f(c)\}_{c=1}^{\infty}$ by the help of Nagell [2], $\S 2$. Evidently, (I) the 
degrees of the irreducible factors of $f(X)$ are at most 2; (II) the discriminant of $f(X)$ is not zero. For a prime number $p$, there is a natural number $c$ such that $b^{6} f(c)=\left(b^{6} c \pm 1\right)^{6}-1 \not \equiv 0\left(\bmod p^{2}\right)$ if $b \neq \equiv 0(\bmod p)$, and there is a $c^{\prime}$ such that $f\left(c^{\prime}\right) \equiv 6 c^{\prime} \not \equiv 0\left(\bmod p^{2}\right)$ if $b \equiv 0(\bmod p)$. This implies that (IV) there is no prime number $p$ such that $f(c) \equiv 0\left(\bmod p^{2}\right)$ for all natural numbers $c=1,2, \cdots$. Now let us assume that $b$ is prime to 6 . Then (III) the polynomial $f(X)$ is primitive. From (I), (II), (III) and (IV), we can apply [2], §2, I, and find infinitely many square free natural numbers in $\{f(c)\}_{c=1}^{\infty}$. When $b$ is not prime to 6 , we apply Nagell's result to $(1 / 2) f(2 X+1)$, $(1 / 3) f(3 X+1)$ or $1 / 6 f(6 X+1)$ in a similar but slightly different manner from the above in order to prove the assertion.

REMARK 4. Stender has given in [10] an explicit formula for the fundamental units of $Q(\sqrt[6]{M})$, where $M=N^{6} \pm n(>1)$ with natural numbers $N$ and $n$ such that $n$ is 6 th power free and divides $N^{5}$, assuming that $\left(N^{6} / n\right) \pm 1$ or $N^{6} / n$ is square free. We will see that Theorem 1 is contained in his result only if $b=1$. Let $n=p_{1}^{v_{1}} \ldots$ $p_{s}^{v_{s}}\left(v_{j}=1,2, \cdots, 5\right)$ with distinct prime numbers $p_{1}, \cdots, p_{s}$. Write $\left(N^{6} / n\right) \pm 1=m x^{6}$ with natural numbers $m$ and $x$, where $m$ is 6 th power free. Put $m^{\prime}=\left(p_{1} \cdots p_{s}\right)^{6} / n$; then $m^{\prime}$ is also 6 th power free. When $M=N^{6}+n$, the diophantine equation $m X^{6}-m^{\prime} Y^{6}=1$ belongs to the field $\boldsymbol{Q}(\sqrt[6]{\bar{M}})$ in the sense of [10], Definition 1, and has a solution $(X, Y)=\left(x, N / p_{1} \cdots p_{s}\right)$ (see also [10], Satz 10). On the other hand, the equation $X^{6}-d Y^{6}=1$ belongs to $K$ and has a solution $(X, Y)=(a, b)$. Suppose $K=\boldsymbol{Q}(\sqrt[6]{M})$; then it follows from [10], Satz 7 that

$$
m=1, \quad m^{\prime}=d, \quad x=a, \quad N / p_{1} \cdots p_{s}=b .
$$

Then $\left(N^{6} / n\right)+1=x^{6}$ cannot be square free. If $N^{6} / n$ is square free, $n=N^{5}$ and $N$ is square free. Therefore $N=p_{1} \cdots p_{s}$, i.e., $b=1$. When $M=N^{6}-n$, we similarly obtain

$$
m=d, \quad m^{\prime}=1, \quad x=b, \quad N / p_{1} \cdots p_{s}=a,
$$

if $K=Q(\sqrt[6]{M})$. If $\left(N^{6} / n\right)-1$ is square free, then $x=b=1$. If $N^{6} / n$ is square free, then $n=N^{5}=1$, and this is a contradiction. Thus, we have seen that Theorem 1 is not contained in Satz 22 of Stender [10] if $b>1$.

4. Real Minkowski unit. Let $K=\boldsymbol{Q}(\theta)(\theta=\sqrt[6]{d)}$ be a real pure sextic field, and $L=K(\zeta)$ its galois closure, where $\zeta=\exp (2 \pi \sqrt{-1} / 3)$. According to A. Brumer [1], we say a unit $\xi$ of $L$ is a Minkowski unit of $L$ if we can take 4 conjugates $\xi^{(1)}, \cdots, \xi^{(4)}$ of $\xi$ such that 
$\xi, \xi^{(1)}, \cdots, \xi^{(4)}$ form a set of fundamental units of $L$. The galois group of $L$ over $\boldsymbol{Q}$ is generated by the two automorphisms $\sigma$ and $\tau$ which satisfy

$$
\theta^{\sigma}=-\zeta \theta, \quad \theta^{\tau}=\theta ; \quad \zeta^{\sigma}=\zeta, \quad \zeta^{\tau}=\zeta^{-1} .
$$

The defining relations of $\sigma$ and $\tau$ are $\sigma^{6}=\tau^{2}=(\sigma \tau)^{2}=1$. We will give an example of a real Minkowski unit of the non-abelian, galois, totally imaginary field $L$. Since $K$ is a maximal real subfield of $L$, it suffices to find a unit $\xi$ of $K$ such that $\xi, \xi^{\sigma}, \cdots, \xi^{\sigma^{4}}$ form a set of fundamental units of $L$. Now let $d, a$ and $K$ be as in $\S 3$. Assume $d>1$ and is square free. Using the same notation as before, we first study the subfields of $L$.

Proposition 4. The assumptions being as above, (i) $\xi_{1}^{1+\sigma^{2}+\sigma^{4}}$ is a fundamental unit of $K_{2}(\zeta)$, (ii) $\xi_{1}^{1+\sigma^{3}}, \xi_{1}^{\left(1+\sigma^{3}\right) \sigma}$ form a set of fundamental units of $K_{3}(\zeta)$, (iii) $\xi_{1}^{o+a^{2}}, \xi_{1}^{a^{4}+\sigma^{5}}$ form a set of fundamental units of the fixed field $F=\boldsymbol{Q}(\sqrt[6]{-27 d})$ of $\sigma^{3} \tau$.

Proof. (i) On acconut of (6), $\eta_{2}=1 / \xi_{1} \xi_{3}=a^{3}+b^{3} \theta^{3}$ is a fundamental unit of $K_{2}$. Suppose that $\eta_{2}$ is not a fundamental unit of $K_{2}(\zeta)$. Then since $d \neq 3$, it follows from S.-K. Kuroda [4], Satz 14, that $3 \eta_{2}=\alpha^{2}$ with an integer $\alpha$ of $K_{2}$ Since $d \not \equiv 1(\bmod 4)$, we have $\alpha=x+y \theta^{3}$ with rational integers $x$ and $y$. Therefore

$$
3\left(a^{3}+b^{3} \theta^{3}\right)=\left(x+y \theta^{3}\right)^{2} .
$$

Comparing the coefficients and taking the norms of both sides of this equation, we see

$$
3 a^{3}=x^{2}+d y^{2}, \quad 9=\left(x^{2}-d y^{2}\right)^{2} .
$$

This leads us to a contradiction after an easy calculation using the fact that $d$ is square free. Hence $\eta_{2}=1 / \xi_{1} \xi_{2}=\xi_{1}^{-\left(1+\sigma^{2}+\sigma^{4}\right)}$ is a fundamental unit of $K_{2}(\zeta)$. (ii) On account of (6), $\eta_{3}^{-1}=\xi_{1} \xi_{2}=a^{2}-b^{2} \theta^{2}$ is a fundamental unit of $K_{3}$. Suppose that $\eta_{3}^{-1}$ and $\eta_{3}^{-\sigma}$ does not form a set of fundamental units of $K_{3}(\zeta)$. Then we have

$$
\beta^{1+\tau}=\operatorname{Tr}_{Q}^{K_{3}}\left(1+\eta_{3}^{-1}+\eta_{3}\right)=3\left(a^{4}+a^{2}+1\right)
$$

with an integer $\beta$ of $\boldsymbol{Q}(\zeta)$ such that $(\gamma / \beta)+(\gamma / \beta)^{\tau}$, where $\gamma=1+$ $\eta_{3}^{-1}+\eta_{3}^{-(1+\sigma)}$, is an integer of $K_{3}$ (see K. Iimura [3], Theorem 1 and Proposition). Put $\beta=x+y \zeta$ with rational integers $x$ and $y$; then we can compute $(\gamma / \beta)+(\gamma / \beta)^{\tau}$ by $(8)$, and see that the coefficient of $\theta^{4}$ is equal to $(x+y) b^{4} / 3\left(a^{4}+a^{4}+1\right)$. Since $d$ is square free, it follows that $(x+y) b^{4} /\left(a^{4}+a^{2}+1\right)$ is a rational integer. By (2), $b$ and $a^{4}+$ $a^{2}+1$ have no common divisor except 3. Moreover, since $(x+y)^{2}-$ 
$3 x y=3\left(a^{4}+a^{2}+1\right)$ by (8), $x+y$ and $a^{4}+a^{2}+1$ have no common divisor except 3 , because $a^{4}+a^{2}+1$ is square free as a divisor of $d$. Therefore $a^{4}+a^{2}+1=3$, i.e., $a=1$ follows. This is a contradiction. Hence $\eta_{3}^{-1}=\xi_{1} \xi_{2}=\xi_{1}^{1+\sigma^{3}}$ and $\eta_{3}^{-\sigma}=\xi_{1}^{\left(1+\sigma^{3}\right) \sigma}$ form a set of fundamental units of $K_{3}(\zeta)$. (iii) Let $H^{\prime}$ be the subgroup of the group $E_{F}$ of units of $F$ given by

$$
H^{\prime}=\left\{\xi \in E_{F} \mid \xi^{1+\tau}=1\right\} .
$$

Then $H^{\prime}$ is generated by a unit $\varepsilon_{2}$ and the roots of unity in $F$ (see [10], $\S 4$, II). It is easy to see that $\xi_{1}^{\left(\sigma+\sigma^{2}\right)(1+\tau)}=\xi_{1}^{-\left(1+\sigma^{3}\right)}=\eta_{3}$, and that $\xi_{1}^{\left(\sigma+\sigma^{2}\right)\left(1-\sigma^{3}\right)(1+\tau)}=1$. Therefore $\xi_{1}^{\left(\sigma+\sigma^{2}\right)\left(1-\sigma^{3}\right)}=\omega \varepsilon_{2}^{n}$ with a rational integer $n$ and a root of unity $\omega$. Applying $\sigma+\sigma^{2}$ to both sides, we obtain $\xi_{1}^{-1+3 \sigma^{3}+2\left(\sigma^{2}+\sigma^{4}\right)}=\omega^{\sigma+\sigma^{2}} \varepsilon_{2}^{n\left(\sigma+\sigma^{2}\right)}$. Since $F$ is the fixed field of $\sigma^{3} \tau, \varepsilon_{2}^{\sigma+\sigma^{2}}$ is a unit of $K$, and hence $\omega^{\sigma+\sigma^{2}}$ also belongs to $K$. Recall that $\xi_{1}, \xi_{1}^{\sigma^{3}}$, $\xi_{1}^{\sigma^{2}+\sigma^{4}}$ form a set of fundamental units of $K$ by Theorem 1 . Consequently $n= \pm 1$, and $\xi_{1}^{\left(\sigma+\sigma^{2}\right)\left(1-\sigma^{3}\right)}$ and the roots of unity of $F$ generate $H^{\prime}$. As we have seen above, $\xi_{1}^{\left(\sigma+\sigma^{2}\right)(1+\tau)}=\eta_{3}$. According to [10], Satz $24, \hat{\xi}_{1}^{\left(\sigma+\sigma^{2}\right)\left(1-\sigma^{3}\right)}$ and $\xi_{1}^{\left(\sigma+\sigma^{2}\right)}$ form a set of fundamental units of $F$. This completes the proof of (iii).

THEOREM 2. Under the same assumptions as in Theorem 1, the galois closure $L=K(\zeta)$ of $K$ has a real Minkowski unit $\xi_{1}=a-b \theta$.

Proof. Let $E^{\prime}$ be the subgroup of the group of units of $L$ which is generated by all the units of $K, K^{\sigma^{2}}, K^{\sigma^{4}}$ and $K_{2}(\zeta)$. Then for every unit $\xi$ of $L, \xi^{3}=\xi^{1+\tau} \xi^{1+\sigma^{2} \tau} \xi^{1+\sigma^{4}} \tau \xi^{-\tau\left(1+\sigma^{2}+\sigma^{4}\right)}$ belongs to $E^{\prime}$. On the other hand, by Proposition 4(i) and Theorem 1, $E^{\prime}$ is generated by the roots of unity in $L$ and $\xi_{1}, \xi_{1}^{\sigma}, \cdots, \xi_{1}^{\sigma^{4}}$. Hence

$$
\xi^{3}=\omega \xi_{1}^{n_{0}+n_{1} \sigma+\cdots+n_{4} \sigma^{4}},
$$

where $\omega$ is a root of unity and $n_{0}, n_{1}, \cdots, n_{4}$ are rational integers. By applying $1+\tau, 1+\sigma^{3} \tau$ and $1+\sigma^{3}$ to both sides, we get

$$
\begin{aligned}
& \xi^{3(1+\tau)}=\xi_{1}^{\left(2 n_{0}-n_{1}\right)} \xi_{1}^{\left(2 n_{3}-n_{1}\right) \sigma^{3}} \xi_{1}^{\left(\left(n_{2}+n_{4}-n_{1}\right)\left(\sigma^{2}+\sigma^{4}\right)\right.}, \\
& \xi^{3\left(1+\sigma^{3} \tau\right)}=\omega^{\prime} \xi_{1}^{\left(n_{1}+n_{2}-n_{0}-n_{3}\right)\left(\sigma+\sigma^{2}\right)} \xi_{1}^{\left(n_{4}-n_{0}-n_{3}\right)\left(\sigma^{4}+\sigma^{5}\right)}, \\
& \xi^{3\left(1+\sigma^{2}\right)}=\omega^{\prime \prime} \xi_{1}^{\left(n_{0}+n_{3}-n_{1}-n_{4}\right)\left(1+\sigma^{3}\right)} \xi_{1}^{\left(n_{2}-n_{1}-n_{4}\right)\left(1+\sigma^{3}\right) \sigma},
\end{aligned}
$$

where $\omega^{\prime}$ and $\omega^{\prime \prime}$ are roots of unity. By Theorem 1 and Proposition 4(ii) and (iii), we see that $n_{0} \equiv n_{1} \equiv \cdots \equiv n_{4} \equiv 0(\bmod 3)$. This implies that $\xi^{3}$ is already a cube in $E^{\prime}$ modulo the roots of unity, and hence $\xi$ belongs to $E^{\prime}$. This shows that $E^{\prime}$ is the group of all units of $L$, and that $\xi_{1}$ is a real Minkowski unit of $L$.

Concluding REMaRK. Stender's method is based on the group 
of relative units of a non-galois number field which has proper subfields. We can generalize this to a field whose galois closure is a dihedral extension over $\boldsymbol{Q}$ (see [7]).

\section{REFERENCES}

1. A. Brumer, On the group of units of an absolutely cyclic number field of prime degree, J. Math. Soc. Japan, 21 (1969), 357-358.

2. G. Degert, Über die Bestimmung der Grundeinheit gewisser reelquadratischer Zahlkörper, Abh. Math. Sem. Hamburg, 22 (1958), 92-97.

3. K. Iimura, On the unit groups of certain sextic number fields, (to apper in Abh. Math. Sem. Hamburg, 50).

4. S. -K. Kuroda, Über den Dirichletschen Körper, J. Fac. Sci. Univ. Tokyo, 4 (1943), 383-406.

5. T. Nagell, Zur Arithmetik der Polynome, Abh. Math. Sem. Hamburg, 1 (1922), 174-194.

6. T. Nagell, Solution complète de quelques équations cubiques à deux indéterminées, J. Math. Pures Appl., 4 (1925), 209-270.

7. K. Nakamula, On the group of units of a nongalois quartic or sextic number field, (to appear).

8. H. -J. Stender, Über die Einheitengruppe der reinen algebraischen Zahlkörpern sechsten Grades, J. Reine Angew. Math., 268/269 (1974), 78-93.

9. — Eine Formel für Grundeinheiten in reinen algebraischen Zahlkörpern dritten, vierten und sechsten Grades, J. Numb. Th., 7 (1975), 235-250.

10. - Lösbare Gleichungen $a x^{n}-b y^{n}=c$ und Grundeinheiten für einige algebraische Zahlkörper vom Grade $n, n=3,4,6$, J. Reine Angew. Math., 290 (1977), $24-62$.

11. H. Yokoi, On real quadratic fields containing units with norm -1, Nagoya Math. J., 33 (1968), 139-152.

12. - On the fundamental unit of real quadratic fields with norm 1, J. Number Theory, 2 (1970), 106-115.

13. - The diophantine equation $x^{3}+d y^{3}=1$ and the fundamental unit of a real pure cubic field $\boldsymbol{Q}(\sqrt[3]{d})$, J. Reine Angew. Math., 268/269 (1974), 174-179.

Received June 8, 1977 and in revised form December 21, 1978.

Tokyo Metropolitan University

2-1-1 Fukazawa, Setagaya-Ku

TOKYO, JAPAN 



\title{
PACIFIC JOURNAL OF MATHEMATICS
}

\section{EDITORS}

DONALD BABBITT (Managing Editor)

University of California

Los Angeles, CA 90024

\section{HUGO RossI}

University of Utah

Salt Lake City, UT 84112

C. C. MOORE and ANDREW OGG

University of California

Berkeley, CA 94720

\section{J. DUGUNDJI}

Department of Mathematics University of Southern California Los Angeles, CA 90007

R. FINN and J. Milgram

Stanford University Stanford, CA 94305

\section{ASSOCIATE EDITORS}
E. F. BECKENBACH
B. H. NeUmanN
F. WOLF
K. YosHIDA

\section{SUPPORTING INSTITUTIONS}

\author{
UNIVERSITY OF BRITISH COLUMBIA \\ CALIFORNIA INSTITUTE OF TECHNOLOGY \\ UNIVERSITY OF CALIFORNIA \\ MONTANA STATE UNIVERSITY \\ UNIVERSITY OF NEVADA, RENO \\ NEW MEXICO STATE UNIVERSITY \\ OREGON STATE UNIVERSITY \\ UNIVERSITY OF OREGON
}

\author{
UNIVERSITY OF SOUTHERN CALIFORNIA \\ STANFORD UNIVERSITY \\ UNIVERSITY OF HAWAII \\ UNIVERSITY OF TOKYO \\ UNIVERSITY OF UTAH \\ WASHINGTON STATE UNIVERSITY \\ UNIVERSITY OF WASHINGTON
}

The Supporting Institutions listed above contribute to the cost of publication of this Journal, but they are not owners or publishers and have no responsibility for its content or policies.

Mathematical papers intended for publication in the Pacific Journal of Mathematics should be in typed form or offset-reproduced, (not dittoed), double spaced with large margins. Please do not use built up fractions in the text of the manuscript. However, you may use them in the displayed equations. Underline Greek letters in red, German in green, and script in blue. The first paragraph or two must be capable of being used separately as a synopsis of the entire paper. Please propose a heading for the odd numbered pages of less than 35 characters. Manuscripts, in triplicate, may be sent to any one of the editors. Please classify according to the scheme of Math. Reviews, Index to Vol. 39. Supply name and address of author to whom proofs should be sent. All other communications should be addressed to the managing editor, or Elaine Barth, University of California, Los Angeles, California, 90024.

50 reprints to each author are provided free for each article, only if page charges have been substantially paid. Additional copies may be obtained at cost in multiples of 50 .

The Pacific Journal of Mathematics is issued monthly as of January 1966. Regular subscription rate: $\$ 84.00$ a year (6 Vols., 12 issues). Special rate: $\$ 42.00$ a year to individual members of supporting institutions.

Subscriptions, orders for numbers issued in the last three calendar years, and changes of address should be sent to Pacific Journal of Mathematics, P.O. Box 969, Carmel Valley, CA 93924, U.S.A. Older back numbers obtainable from Kraus Periodicals Co., Route 100, Millwood, NY 10546.

PUBLISHED BY PACIFIC JOURNAL OF MATHEMATICS, A NON-PROFIT CORPORATION

Printed at Kokusai Bunken Insatsusha (International Academic Printing Co., Ltd.). 8-8, 3-chome, Takadanobaba, Shinjuku-ku, Tokyo 160, Japan.

Copyright (C) 1979 by Pacific Journal of Mathematics Manufactured and first issued in Japan 


\section{Pacific Journal of Mathematics}

\section{Vol. 83, No. $2 \quad$ April, 1979}

Patrick Robert Ahern, On a theorem of Hayman concerning the derivative of a

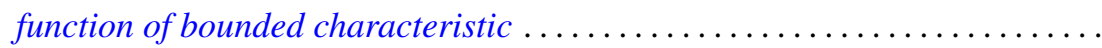

Walter Allegretto, Finiteness of lower spectra of a class of higher order elliptic

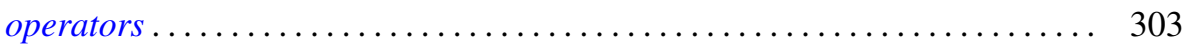

Leonard Asimow, Superharmonic interpolation in subspaces of $C_{c}(X) \ldots \ldots 11$

Steven F. Bellenot, An anti-open mapping theorem for Fréchet spaces . . . . . . . 325

B. J. Day, Locale geometry. . . . . . . . . . . . . . . . . . . . . . . . . . 333

John Erik Fornaess and Steven Krantz, Continuously varying peaking

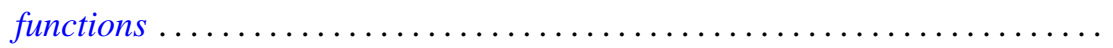

Joseph Leonide Gerver, Long walks in the plane with few collinear points ......

Joseph Leonide Gerver and Lawrence Thom Ramsey, On certain sequences of

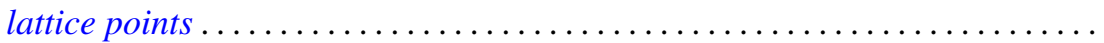

John R. Graef, Yuichi Kitamura, Takaŝi Kusano, Hiroshi Onose and Paul Winton

Spikes, On the nonoscillation of perturbed functional-differential

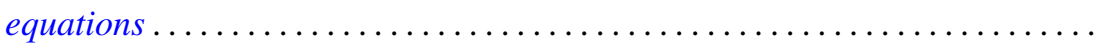

James A. Huckaba and James M. Keller, Annihilation of ideals in commutative

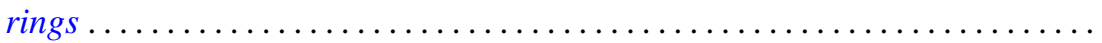

Anzelm Iwanik, Norm attaining operators on Lebesgue spaces . . . . . . . . . . .

Surjit Singh Khurana, Pointwise compactness and measurability . . . .......... 387

Charles Philip Lanski, Commutation with skew elements in rings with

involution.

Hugh Bardeen Maynard, A Radon-Nikodým theorem for finitely additive bounded

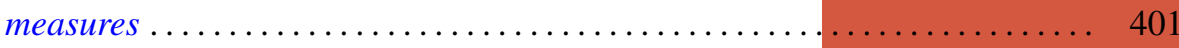

Kevin Mor McCrimmon, Peirce ideals in Jordan triple systems ..

Sam Bernard Nadler, Jr., Joseph E. Quinn and N. Stavrakas, Hyperspaces of compact convex sets.

Ken Nakamula, An explicit formula for the fundamental units of a real pure

sextic number field and its Galois closure ............

Vassili Nestoridis, Inner functions invariant connected components . .

Vladimir I. Oliker, On compact submanifolds with nondegenerate parallel

normal vector fields.

Lex Gerard Oversteegen, Fans and embeddings in the plane.

Shlomo Reisner, On Banach spaces having the property G.L

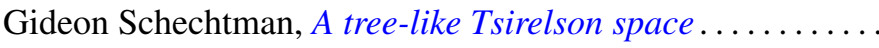

Helga Schirmer, Fix-finite homotopies . . . . . . . . . . . .

Jeffrey D. Vaaler, A geometric inequality with applications to linear forms . .

William Jennings Wickless, $T$ as an $\mathscr{G}$ submodule of $G$.....

Kenneth S. Williams, The class number of $Q(\sqrt{-p})$ modulo 4 , for $p \equiv 3$ (

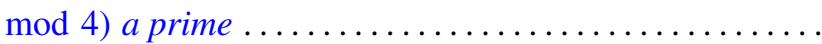

